\title{
The influence of the equidistance tendency on depth shifts resulting from an interocular delay in stimulation '
}

EUGENE R. WIST

FRANKLIN AND MARSHALL COLLEGE

An earlier study showed that the introduction of an interocular delay in presentation to the two eyes of a briefly viewed, haploscopically presented object resulted in a rearward shift in the depth position of this object. The present study was designed to determine whether an equidistance tendency (ET) which opposed this direction of shift could reverse or modify the degree of shift. It was found that an opposing ET did attenuate the degree of depth shift, but did not reverse the direction of shift. Implications of this finding for the explanation of the depth shift phenomena are discussed.

In an earlier study (Wist \& Gogel, 1966) it was found that the introduction of an interocular delay in presentation to the two eyes of a briefly viewed haploscopically presented test object resulted in a shift in the depth position of this object. As the interocular delay interval (IDI) was increased from 0 to $96 \mathrm{msec}$, the haploscopically viewed comparison object appeared increasingly behind the standard. In other words, there was a shift in the perceived depth position of the variable stimulus object, the magnitude of which was a function of IDI.

Two related questions were raised by this experiment. Why does the interocular delay interval lead to any shift in the perceived position of the haploscopic variable stimulus, and why is this shift in depth away from rather than toward the subject?

In attempting to account for our results, we described a temporal continuum between alternate monocular stimulation at long interocular delay intervals, and binocular stimulation at simultaneous or nearly simultaneous stimulation of the two eyes. We considered the hypothesis that as interocular delay interval exceeds some critical threshold value, there is a change from binocular fusion to alternate monocular perception of the stimulus in each eye. When this happens, it would be consistent with Panum's Limiting Case (Ogle, 1962) for the monocular object, the variable stimulus, to appear farther away than the standard object. This interpretation, which we saw as the simplest one, attributes the depth shift to a breakdown in binocular fusion.

We discussed the equidistance tenciency (ET) as another variable which ought to affect the perceived position of the variable stimulus (Gogel, 1965). According to the ET, two or more objects presented simultaneously in a visually reduced situation will tend to appear at the same distance. The extent to which they are seen as not equidistant is determined by the number and strength of depth cues remaining in the situation.

Accordingly, a pilot study was performed with monocular presentation of the variable stimulus to determine whether it might be possible to oppose the panum effect with the ET. We wished to set up a situation in which the monozular position of the variable stimulus was in front of rather than behind the standard stimulus. If the direction of the shift in depth that occurs with increased interocular delay interval is determined by the monocular depth position of the variable stimulus, it ought to be possible to change the direction of the depth shift by opposing the ET to Panum's Limiting case. If a situation is arranged in which the ET operates to make the haploscopic object appear in front of the standard when it is monocular, then as the interocular delay interval is increased under this condition, the variable stimulus should shift in perceived depth location in the direction of the subject. When the ET is not in opposition to Panum's Limiting case, the perceived position of this stimulus ought to shift away from $S$ as in the earlier study (Wist \& Gogel, 1966).

The results of this pilot study supported the above rationale and therefore made it feasible to carry out the present investigation. If the shift in perceived depth location of the haploscopically viewed variable stimulus is the result of this stimulus becoming increasingly monocular as the IDI increases, and if Panum's Limiting Case and the ET are involved in determining the monocular depth location of this stimulus, then it ought to be possible to produce both forward and rearward depth shifts depending upon whether the ET opposes (forward shift) or is consistent with Panum's Limiting Case (rearward shift).

\section{HETHOD}

\section{Subjects}

Eight paid college students, four men and four women were employed as Ss. Each had considerable experience in four previous experiments with the tasks required in the present study. All had a corrected visual acuity of at least $20 / 20$ and a stereoacuity as measured with the diagnostic series of the Keystone Telebinocular of at least $19 \mathrm{sec}$ arc. 
A
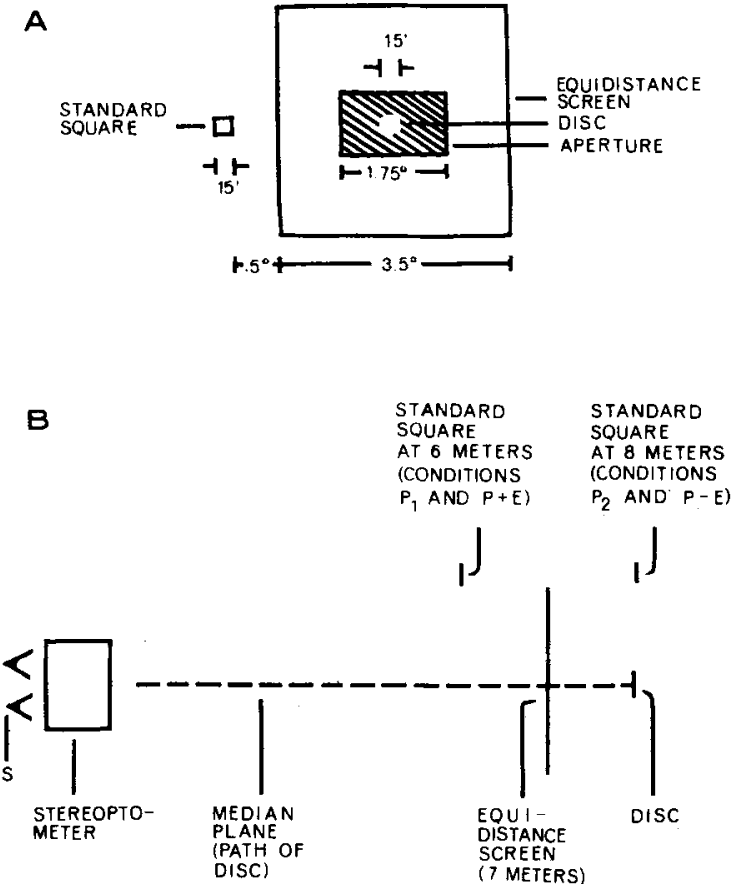

Fig. 1A. Frontal view of the stimulus display with angular dimensions.

Fig. 1B. Overhead view of visual alley showing displacement in depth of the stimuli in $A$. (Not drawn to scale)

\section{Apparafus}

To produce the IDIs, a flash generator (Roush \& Hamburger, 1948) was used to drive two Sylvania R1131C glow modulator tubes which transilluminated the two reticles of a stereoptometer, a stereoscopic range finder of unit base and unit magnification (Harker, 1955). The reticle images were registered upon the eye by the parallel optical systems of this instrument, so that $S$ saw a fused image of the two disc shaped reticles projected at optical infinity. This same system was used in the earlier study (Wist \& Gogel, 1966).

For simultaneous binocular stimulation, both reticles were illuminated simultaneously. For monocular stimulation, either the left eye or right eye reticle could be illuminated. An interocular delay in stimulation was produced by presenting a $4 \mathrm{msec}$ flash of the glow modulator for the left eye, followed after a delay of 16, 32, or $64 \mathrm{msec}$ by a $4 \mathrm{msec}$ flash of the glow modulator tube for the right eye. A repetition interval, as measured from flash onsets, of $600 \mathrm{msec}$ separated successive flash pairs. The earlier study demonstrated that flash pairs separated by this period were independent in terms of their effect on the perceived depth position of the disc (Wist \& Gogel, 1966).

Figure 1a is a frontal view of the visual display and contains the angular dimensions of all stimuli. The standard square and the equidistance screen were binocularly observed and continuously transilluminated. They consisted of light boxes containing cool white $40 \mathrm{~W}$ fluorescent tubes. Milk Lucite filters were used to reduce their intensity to $1.5 \mathrm{ft}-\mathrm{L}$. The disc was generated by the optical system deseribed above and was transilluminated by means of the flash generator circuit. It appeared intermittently at a repetition interval of $600 \mathrm{msec}$. At the beginning of each session, $S$ matched the luminance of the disc 10 times for each eye independently to the luminance of the equidistance screen. The mean of these 10 adjustments was used to set the luminance of each disc for the remainder of the session.

Figure $1 \mathrm{~b}$ is an overhead view of the visual display. The equidistance screen was located at $7 \mathrm{~m}$ from S's eyes. The dashed line indicated the median plane along which the disc was perceived to move when the control on the stereoptometer which changed the vergence angle between the two optical systems was manipulated by $S$.

\section{Depth shitt task}

S's task under all conditions was to adjust the position in depth of the disc by changing the vergence angle between the two optical systems of the stereoptometer until the disc appeared equidistant to the standard square located on the left at either 6 or $8 \mathrm{~m}$. If the disc appeared to be behind the standard square when the retinal disparity between these two objects was zero, then $S$ would have to increase the vergence angle to the disc (make the disc move toward him) in order to make it appear equidistant to the standard. The opposite would, of course, be the case, if the disc appeared to be in front of the standard when the retinal disparity between them was zero. The method of adjustment was used. Each $S$ made five consecutive adjustments under each of the experimental conditions. After each adjustment, $E$ changed the vergence angle of the disc, increasing it after half of the trials and increasing it on the remainder in a counterbalanced order. For all adjustments, fixation was maintained on the standard square.

\section{Experimental Design}

Each of the eight Ss ran under four conditions, one per session. The order of conditions was randomized. Within each session, each $S$ made five adjustments of the depth position of the disc under four different interocular delay (IDI) intervals, $0,16,32$, and 64 msec, presented in a random order. Table 1 summarizes the stimulus arrangement and the predicted

Table 1. Experimental Design

\begin{tabular}{|c|c|c|c|c|}
\hline \multirow[t]{2}{*}{ Condition } & \multirow[t]{2}{*}{$\begin{array}{l}\text { Equidistance } \\
\text { Screen at } 7 \mathrm{~m} \text {. }\end{array}$} & \multirow[t]{2}{*}{$\begin{array}{c}\text { Distance of } \\
\text { Standard Square }\end{array}$} & \multicolumn{2}{|c|}{$\begin{array}{l}\text { Predicted Shifts } \\
\text { on basis of: }\end{array}$} \\
\hline & & & & \\
\hline & & & Back & Back \\
\hline & $A b$ & $6 n$ & -- & Bock \\
\hline & Present & $8 \mathrm{~m}$. & Forward & Back \\
\hline & Absent & $8 \mathrm{~m}$. & -- & Back \\
\hline
\end{tabular}

*Equidistance Tendency, ** Panum's Limiting Case 


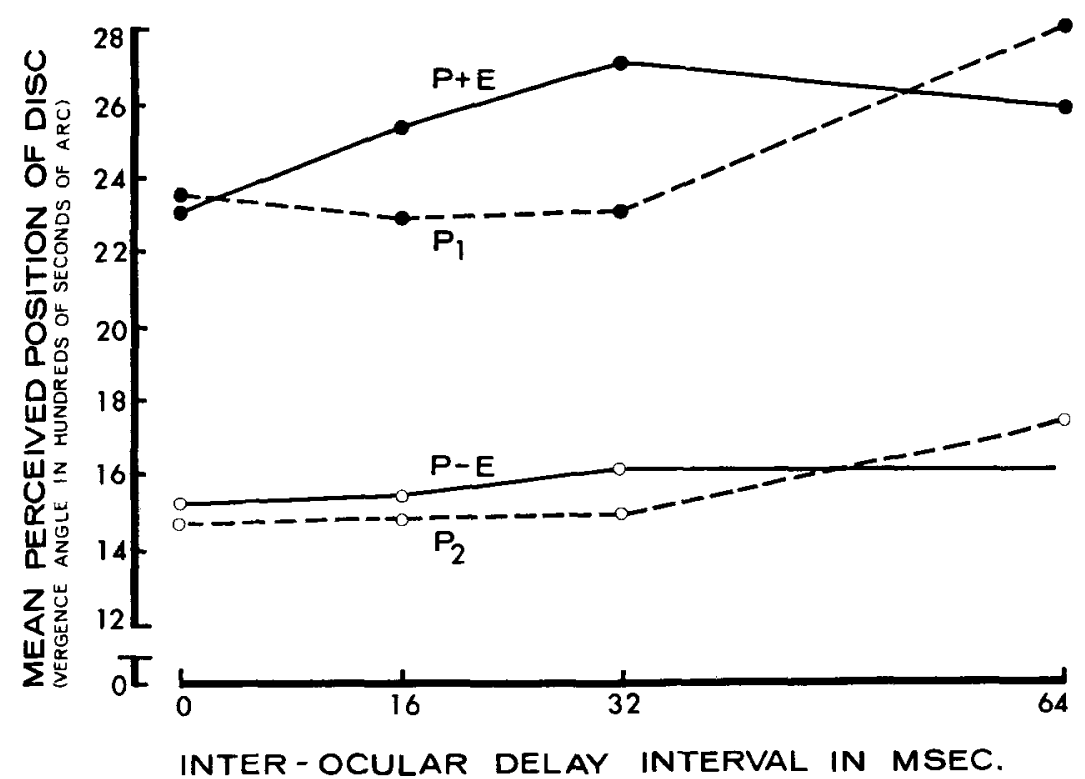

Fig. 2. Mean perceived position of the disc as a function of inter-ocular delay interval and viewing conditions. Larger vergence angles mean that the disc appeared farther away from $\mathrm{S}$. shifts in the depth position of the disc for each of the four conditions. For Condition E+P, the standard square to which the $S$ adjusted the depth position of the disc was in front of the equidistance screen. Therefore, both the ET and Panum's Limiting Case would predict a backward (away from $S$ ) shift in the perceived position of the disc. For Condition P-E, the ET and Panum's Limiting Case are in opposition. Since the equidistance screen was in front of the standard square for this condition, on the basis of the ET a forward shift in the disc position with increasing IDI would be predicted. Thus, for this condition, Panum's Limiting Case and the ET are in opposition. Conditions $P_{1}$ and $P_{2}$ were control conditions. The equidistance screen was not present for these conditions. Consequently, Panum's Limiting Case was neither supported as in E+P nor opposed as in P-E. 2

\section{Light Adapration}

At the beginning of each session, $\mathrm{S}$ received $10 \mathrm{~min}$ of light adaptation to a homogenous $1.5 \mathrm{ft}-\mathrm{L}$ screen, $50 \mathrm{deg}$ high and $80 \mathrm{deg}$ wide. Adaptation also occurred after each block of five adjustments of the disc with the length of the adaptation period being either the time required for the five adjusts or $3 \mathrm{~min}$ whichever was shorter.

\section{RESULTS}

The results of the experiment are presented in Fig. 2. This figure contains the data for all eight Ss. Each point represents the mean of 40 adjustments of the disc, five from each of the eight Ss. Ignoring the $64 \mathrm{msec}$ IDI for the moment, it can be seen that the $\mathrm{P}+\mathrm{E}$ condition resulted in a greater rearward shift in the perceived position of the disc than the $P_{1}$ condition as predicted. Furthermore, the slope of the $\mathrm{P}+\mathrm{E}$ function is steeper than that of the P-E function as would be expected, since in the latter condition the ET and Panum's Limiting Case were in opposition. An analysis of variance performed on these data resulted in $F$ ratios significant at the $p<.01$ level for these differences.

The crossing over of the functions at $64 \mathrm{msec}$ was the result of the unusual function obtained for one $S$ for Condition $P_{1}$ and for another $S$ for Condition $P-E$. If their data were excluded from the graph these functions would not cross over. An earlier study (Wist \& Gogel, 1966) has shown that the standard deviation of adjustments for a $64 \mathrm{msec}$ IDI is about twice that of a $32 \mathrm{msec}$ IDI. Thus, one would expect to obtain less stability for data collected at this $64 \mathrm{msec}$ IDI.

\section{DISCUSSION}

In general, the results of this study seem to support the rationale described in the introduction. When Panum's Limiting Case and the ET both predicted a rearward shift in the perceived depth position of the disc (Condition $P+E$ ) a larger depth shift was obtained than when the predicted directions of depth shift were opposed (Condition P-E). This result suggests an algebraic summation of variables influencing the depth location of the disc. Yet it must be noted that although a strong ET existed between the disc and the equidistance screen in the pilot study where the disc was presented monocularly, so strong that the disc always appeared in the aperture of this screen, no evidence for a forward shift in the perceived position of the disc was found with increasing IDI.

There are at least two possible interpretations of this peculiar fact. One is that the largest IDI, $64 \mathrm{msec}$ 
was not great enough to override the strong Panum effect operating in this situation. A replication of this study is planned using additional IDIs of 96,128 , 256 and $512 \mathrm{msec}$ to test this hypothesis.

The other possibility is that depth shifts can occur within the temporal limits of binocular fusion as well as beyond these limits. That is, depth shifts may occur before the IDI is large enough to result in the successive, monocular perception of the disc by each eye. An obvious way to test this hypothesis would be to obtain for the same Ss depth shifts thresholds and dichoptic two-flash thresholds (Mahneke, 1958), using the latter as a measure of temporal binocular fusion. The former should occur at a shorter IDI than the latter. The results of such an experiment will be reported in a later paper.

\section{References}

Gogel, W. C. Equidistance tendency and its consequences. Psychol. Bull., 1965, 64, 153-163.

Harker, G. S. Instrument for the study of binocular vision. $J$. Opt. Soc. Amer., 1955, 45, 826-828.
Mahneke, A. Foveal discrimination measured with two successive light flashes. Acta Ophthalm., 1958, 36, 3-11.

Ogle, K. N. The optical space sense. In H. Dawson (Ed.), Visual optics and the optical space sense. Vol. 4. New York: Academic Press, 1962.

Roush, R., \& Hamburger, F., Jr. Light-flash generator. Electronics, $1948,21,100-102$.

Wist, E. R., \& Gogel, W. C. The effect of inter-ocular delay and repetition interval on depth perception. Vision Res., 1966, 6, 325-334.

\section{Notes}

1. The data of this study were collected while the author was at the U. S. Army Medical Research Laboratory and constituted a portion of a paper read at the Eastern Psychological Association meetings, April, 1966 in New York City. The author wishes to acknowledge the assistance of Kenneth $\mathrm{J}$. Gall who collected the data and NIH Grant No. NB 05528-02 which made possible the analysis of the data and the preparation of the manuscript.

2. It is true that for all conditions an ET did exist between the standard square and the disc, but because the lateral separation of these two stimuli was large this tendency was weak. (Gogel. 1965) In any case, it was constant for all conditions.

(Accepted for publication October 15, 1967.) 